\title{
RESEARCH HIGHLIGHT GPCR signaling: conformational activation of arrestins
}

\author{
Alem W. Kahsai ${ }^{1}$, Biswaranjan Pani ${ }^{10}$ and Robert J. Lefkowitz ${ }^{1,2,3}$ \\ Cell Research (2018) 28:783-784; https://doi.org/10.1038/s41422-018-0067-x
}

\begin{abstract}
The arrestins, a small family of versatile adapter proteins, play central roles in G-protein coupled receptor (GPCR) desensitization, endocytosis, and signal transduction. Two papers by Latorraca et al. and Eichel et al. published recently in Nature, dissect the independent and concerted mechanisms by which the phosphorylated GPCR tail and transmembrane core conformationally enable arrestins to perform their diverse functions.
\end{abstract}

G-protein coupled receptors (GPCRs), also known as seven transmembrane receptors (7 TMRs) are the largest family of cell surface proteins, with $\sim 800$ members identified in the human genome. ${ }^{1}$ They represent the commonest targets of therapeutic drugs used for the treatment of a wide range of health problems. Their remarkably diverse actions are mediated and modulated by the extracellular stimulus-driven interaction of the receptors with three families of proteins: the heterotrimeric guanine nucleotidebinding $(G)$ proteins, the $G$ protein-coupled receptor kinases (GRKs), and the arrestins. ${ }^{1} \mathrm{G}$ proteins were discovered as the paradigmatic relay switches connecting the receptors to membrane effectors, such as second messenger-generating enzymes. ${ }^{2}$ GRKs and arrestins were discovered as a two-component system, which turns off the receptors. The activated receptors are phosphorylated by the kinases, typically on multiple sites on the cytoplasmic C-terminal tail. This leads to binding of the arrestins, initially to the phosphorylated receptor $C$ tail, and then to the agonist-modified conformation of the 7 TM core. This occludes the G-protein-binding site on the receptor thus diminishing receptor G-protein signaling, a process known as receptor desensitization. ${ }^{1}$ There are four members of the arrestin family including two visual arrestins (arrestins 1 and 4), which are confined to the retina, and two universally expressed non-visual forms, arrestins 2 and 3 , generally referred to as $\beta$-arrestin- 1 and $\beta$-arrestin- 2 . $^{1,3}$

Recently two papers have shed new light on the distinct roles of the phosphorylated receptor C-terminus and the 7 TM core in mediating arrestin interaction with and activation by GPCRs., Protein members of the versatile arrestin family contain two major domains ( $\mathrm{N}$ and $\mathrm{C}$ ), which consist largely of $\beta$-sheets and connecting loops. While initially discovered in the context of receptor desensitization, it was subsequently discovered that $\beta$-arrestins also serve as multifunctional adapters mediating receptor interaction with several components of the clathrincoated pit endocytic machinery, thus mediating receptor internalization and trafficking. ${ }^{1,3,6}$ They also bind to a long list of signaling proteins thus enabling $\beta$-arrestins to serve as signaling intermediates in their own right, acting either independently of or in concert with G-proteins to modulate cellular activities. ${ }^{6}$

The remarkable diversity of functions carried out by arrestins after their interaction with the receptors has engendered much interest in the mechanisms of GPCR- $\beta$-arrestin interactions and how these are translated into specific cellular consequences. Confirming decades-long ideas, recent structural studies of both visual arrestin interaction with rhodopsin and $\beta$-arrestin interaction with other GPCRs, have revealed two types of interactions within receptor-arrestin complexes: one involving the phosphorylated cytoplasmic tail of the GPCR ( $R_{p}$-tail), referred to as the 'tail' interaction, and one involving the transmembrane core of the GPCR, referred to as the 'core' interaction., It has been suggested that these are in turn mediated by two distinct sites on arrestins - a 'phosphorylation sensor' and an 'activation sensor'.

In the past, studies have largely focused on activation of arrestins by the $\mathrm{R}_{\mathrm{p}}$-tail and its functional outcomes, ${ }^{9}$ while the role of the receptor transmembrane core, if any, has remained obscure. However, the two new papers illuminate the distinct roles of both the $R_{p}$-tail and the 7 TM core in mediating arrestin interaction with and activation by GPCRs. ${ }^{4,5}$ Specifically, these studies focus a much brighter light on the mechanisms of arrestin activation by the GPCR core. Using atomic-level molecular dynamic simulations of arrestin and $\beta$-arrestin, Latorraca and colleagues investigated the structural mechanisms by which the $R_{\text {p}}$-tail, and the transmembrane core, in particular, regulate arrestin activation. ${ }^{4}$ In the simulations, the authors used a key conformational change in arrestin that occurs upon activation (a twist of the $\mathrm{C}$ domain of about $20^{\circ}$ relative to the $\mathrm{N}$ domain) as a primary indicator of arrestin activation. ${ }^{7}$ According to current models, the initial step of arrestin activation requires the displacement of the long $C$ tail of arrestin (which is folded back on the $\mathrm{N}$ domain in the inactive conformation) by the $\mathrm{R}_{\mathrm{p}}$-tail of an activated GPCR. The authors show that this displacement of the arrestin $C$ tail leads to prolongation in active state conformations of arrestin, even when not bound to the receptor. This may explain the previously noted curious finding that $\beta$-arrestin remains active and continues signaling following its dissociation from the activating GPCR. $^{10}$

Using the rhodopsin-arrestin structure as a template for their simulations, the authors demonstrate that the receptor core and $\mathrm{R}_{\mathrm{p}}$-tail each individually stabilize active conformations of arrestin (Fig. 1). The simultaneous binding of both regions as occurs with a full-length receptor adds further stabilization. According to their simulations, the key contacts responsible for the core interaction are between the receptor's intracellular loops (ICLs), e.g., ICL-2 and ICL-3, and the C-loop and back loop in the $C$ domain of arrestin, respectively. These computational findings are supported by fluorescence spectroscopic studies on arrestin mutants, which monitor conformational changes both at the $\mathrm{R}_{\mathrm{p}}$-tail interface (the arrestin gate loop) and

\footnotetext{
${ }^{1}$ Department of Medicine, Duke University Medical Center, Durham, NC 27710, USA; ${ }^{2}$ Department of Biochemistry, Duke University Medical Center, Durham, NC 27710, USA and ${ }^{3}$ Howard Hughes Medical Institute, Duke University Medical Center, Durham, NC 27710, USA

Correspondence: Robert J. Lefkowitz (lefko001@receptor-biol.duke.edu)
}

Published online: 20 July 2018 


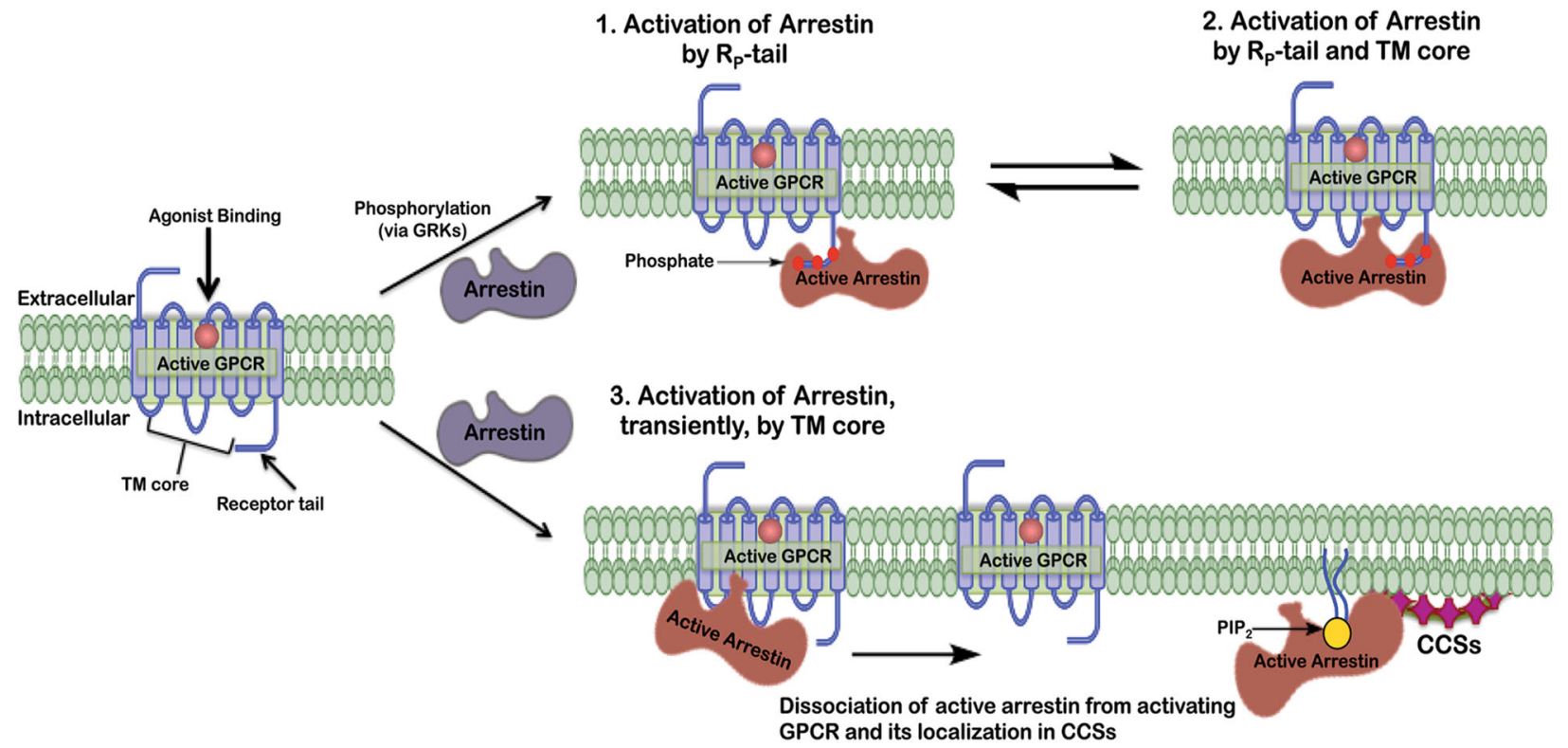

Fig. 1 Proposed modalities of arrestin activation by GPCRs. Two papers ${ }^{4,5}$ shed new light on the mechanisms by which arrestins can be activated by GPCRs. The schematic illustrates three possible binding modes by which GPCRs could activate arrestins involving interactions mediated by: (1) $R_{P}$-tail alone, (2) both the $R_{P}$-tail and the TM core, or (3) the TM core alone. It is proposed that the third activation mode involves transient engagement with the TM core, dissociation from the receptor and subsequent interactions with plasma membrane phosphatidylinositol 4,5-bisphosphate (PIP 2$)$, accumulation in CCSs, and $\beta$-arrestin-dependent signaling in the absence of the activating GPCR

at the core interface (the arrestin $C$ loop) of the protein surfaces.

The findings by Latoracca et al. obtained primarily by computational approaches, are consistent with those of Eichel et al., 5 who, using a variety of cell biological techniques, also demonstrate that transient interactions of the GPCR transmembrane core are sufficient to activate $\beta$-arrestin. Eichel and colleagues had previously shown that, after dissociating from the $\beta_{1}$-adrenergic receptor $\left(\beta_{1} A R\right), \beta$-arrestin- 2 remains in its active state and can transduce $\beta$-arrestin-mediated ERK1/2 signaling from clathrincoated structures (CCSs). ${ }^{10}$ Building on this observation, their current paper delineates the mechanistic basis of this phenomenon. They show that sustained arrestin activation and signaling following dissociation from the activating receptor requires interaction with only the GPCR core, not necessarily the cytoplasmic $R_{p}$-tail. Such activation of $\beta$-arrestin in the plasma membrane involves a series of interactions with membrane phosphoinositides, accumulation in CCSs, and subsequent MAP kinase signaling activity in the absence of the activating receptor. ${ }^{5}$ Activation of $\beta$-arrestin via transient engagement with the GPCR core requires the disruption of a conserved inter-domain interaction network within $\beta$-arrestin. ${ }^{5}$ This network is located proximal to the finger loop, which is known to play a role in maintaining $\beta$-arrestin in its inactive state.

Together, these two papers significantly extend our understanding of how distinct structural elements within GPCRs, particularly the transmembrane core, can activate arrestins to initiate diverse forms of signal transduction in cells. ${ }^{4,5}$ Accumulating evidence has already supported the idea that distinct patterns of phosphorylation on the GPCR C-terminus can trigger specific conformational adjustments in $\beta$-arrestins, leading to specific functional outcomes, the so called 'barcode hypothesis'. ${ }^{11}$ Such multimodal activation of arrestins by the
$\mathrm{R}_{\mathrm{p}}$-tail and core, underscores the intricate and complex choreography that underlies the diverse regulatory functions of $\beta$ arrestin-dependent GPCR activities. This new work also provides a vivid example of how diverse technical approaches can synergistically illuminate important and complex biological phenomena. Going forward, it will be important to learn how generalizable these arrestin activation modes are amongst different GPCRs. Of particular interest is whether there is any relationship between the extent and/or duration of activation of $\beta$-arrestin by the TM core and the previously delineated classification of GPCRs into 'class $A$ ' or 'class $B$ ', which respectively bind $\beta$-arrestins transiently or quite tightly. ${ }^{12}$ Understanding the detailed structural mechanisms of GPCR- $\beta$-arrestin interactions should also provide a foundation for the design of drugs 'biased' toward or away from $\beta$-arrestin-mediated signaling with potentially improved efficacy and fewer side effects.

\section{REFERENCES}

1. Lefkowitz, R. J. Angew. Chem. Int. Ed. Engl. 52, 6366-6378 (2013).

2. Gilman, A. G. Annu. Rev. Biochem. 56, 615-649 (1987).

3. Gurevich, V. V. \& Gurevich, E. V. Pharmacol. Ther. 110, 465-502 (2006).

4. Latorraca, N. R. et al. Nature 557, 452-456 (2018).

5. Eichel, K. et al. Nature 557, 381-386 (2018).

6. Peterson, Y. K. \& Luttrell, L. M. Pharmacol. Rev. 69, 256-297 (2017).

7. Kang, Y. et al. Nature 523, 561-567 (2015).

8. Shukla, A. K. et al. Nature 512, 218-222 (2014).

9. Cahill, T. J. 3rd et al. Proc. Natl Acad. Sci. USA 114, 2562-2567 (2017).

10. Eichel, K., Jullie, D. \& von Zastrow, M. Nat. Cell Biol. 18, 303-310 (2016).

11. Kim, J. et al. Proc. Natl Acad. Sci. USA 102, 1442-1447 (2005).

12. Oakley, R. H., Laporte, S. A., Holt, J. A., Caron, M. G. \& Barak, L. S. J. Biol. Chem. 275, 17201-17210 (2000). 